\title{
Valoración de bienes de uso público en el modelo contable de entidades de gobierno en Colombia. Una aproximación desde los precios hedónicos*
}

Valuation of assets for public use in the accounting model of government entities in Colombia. An approximation from the hedonic prices

Michael Andrés Diaz Jiménez ${ }^{a}$

Universidad Santo Tomás, Colombia

michaeldiazj@usantotomas.edu.co

ORCID: http://orcid.org/0000-0001-8227-0714

Julián Alberto Gutiérrez López

Universidad El Bosque, Colombia

ORCID: http://orcid.org/0000-0001-5296-0853

Ruth Alejandra Patiño Jacinto

Universidad Santo Tomás, Colombia

ORCID: http://orcid.org/0000-0001-6017-7666
DOI: https://doi.org/10.11144/Javeriana.cc19-48.vbup Redalyc: http://www.redalyc.org/articulo.oa? id $=151557795002$

Fecha de recepción: 23 Octubre 2017

Fecha de aprobación: 19 Junio 2018 Fecha de publicación: 20 Diciembre 2018

\section{Resumen:}

Países desarrollados y en vía de desarrollo han modificado su modelo contable como respuesta a los planteamientos de los entes de regulación internacional. Colombia en particular, ha decidido restructurar su Régimen de Contabilidad Pública tomando como referente el modelo internacional. Aunque la Contaduría General de la Nación ha definido una estructura completa, ésta ha presentado algunas dificultades por la falta de herramientas metodológicas para la medición de los bienes de no mercado. El propósito de este artículo es justamente proponer una técnica de medición para actualizar los bienes de uso público, a través de un modelo econométrico que permita determinar la disponibilidad a pagar por el espacio público a partir de precios hedónicos. El resultado alcanzado permite contar con un instrumento metodológico que puede ser utilizado para actualizar el valor de algunos bienes de uso público tanto del distrito capital como de aquellas entidades a nivel nacional e internacional que así lo requieran.

Palabras clave: bienes de uso público, precios hedónicos, valoración económica, NICSP.

\section{Abstract:}

Developed and developing countries have taken the decision to modify their accounting models in response to the approaches proposed by international regulatory bodies. Colombia, in particular, has decided to restructure its Public Accounting Regime taking as reference the international model. Although the Contaduría General de la Nación has established a very complete structure. It has encountered some difficulties in terms of the lack of methodological tools for the measurement of non-market goods. The objective of this paper is to propose a measurement technique to update public assets, by using econometric model for the measurement of public goods with which the availability of public space is determined from hedonic prices. The result achieved will have a methodological tool with a consistent theoretical support. This will be used to update the value of some public assets of the Bogotá capital district, as well as those entities at national and international level that require it. Keywords: public use assets, prices Hedonic, economic valuation, IPSAS.

\section{Introducción}

En los últimos años, numerosos países han modificado su regulación contable atendiendo a las exigencias internacionales. En este proceso, se han implementado los estándares internacionales de contabilidad e información financiera [IFRS] propuestos por el International Accounting Standards Board (IASB), y los

Notas de autor

a Autor de correspondencia. Correo electrónico: michaeldiazj@usantotomas.edu.co 
estándares internacionales de contabilidad para el sector público [IPSAS] planteados por el International Public Sector Accounting Standards Board (IPSASB). En algunas ocasiones se ha optado por el modelo pleno y en otras se contemplan adaptaciones que garantizan la aplicabilidad en los diferentes contextos, esto último ha ocurrido en el sector público en Colombia (Rico \& Díaz, 2017).

La Contaduría General de la Nación (CGN) conformó un nuevo modelo contable público, para el que tomó algunos conceptos de las International Public Sector Accounting Standards (IPSAS) y otras de los IFRS, generando cambios sustanciales en los criterios de reconocimiento, presentación, revelación y en especial, sobre temas de medición en general. Esto implica tener una informacion actualizada y veridica, lo que a su vez recae en labores operativas sobre depuracion y medición de los elementos que componen los estados financieros (Contaduría General de la Nación, 2013).

Producto de los cambios realizados en el modelo contable público se emitió la Resolución No. 533 de 2015, la cual proporciona la reglamentación en materia contable para entidades de gobierno, las cuales tienen por objetivo:

la definición, observancia y ejecución de políticas públicas que buscan incidir en la asignación de bienes y servicios, en la redistribución de la renta y la riqueza, en la estabilidad y el crecimiento económico y en el nivel de bienestar de la sociedad. (Contaduría General de la Nación, 2015d, p. 3)

Dentro de este marco se reglamentan las "Normas para el reconocimiento, medición, revelación y presentación de los hechos económicos" y como caso puntual, lo correspondiente a propiedades, planta y equipo y los bienes de uso público, partidas relevantes para las entidades de gobierno por corresponder a los activos fijos que utilizan las entidades o la comunidad.

De acuerdo con la Resolución 533 de 2015 (Modelo de Entidades de Gobierno), estos activos presentan las características que se exponen en la tabla 1. 
TABLA 1

Características generales de las propiedades, planta y equipo y de los bienes de uso público

\begin{tabular}{|c|c|}
\hline $\begin{array}{c}\text { Clase de activos } \\
\text { no corrientes }\end{array}$ & Características Generales \\
\hline $\begin{array}{l}\text { Propiedades, } \\
\text { planta y equipo }\end{array}$ & $\begin{array}{l}\checkmark \text { Se utilizan para producción o suministro de bienes, prestación de servicios o propósitos } \\
\text { administrativos. } \\
\text { Pueden ser bienes muebles que se entregan en arrendamiento, bienes inmuebles que se } \\
\text { entregan en arrendamiento siempre y cuando sea por un valor inferior al de mercado, bienes } \\
\text { inmuebles con uso futuro indeterminado y plantas productoras (planta viva) que se espera } \\
\text { genere productos agrícolas por más de un período. } \\
\checkmark \text { Se espera usarlos más de un periodo contable y no venderlos dentro de las actividades } \\
\text { ordinarias de la entidad. } \\
\checkmark \text { Se reconocen componentes. } \\
\checkmark \text { La medición inicial se realiza por el costo. } \\
\checkmark \text { La medición posterior se realiza por el costo menos la depreciación acumulada menos el } \\
\text { deterioro acumulado. } \\
\text { Para la transición se puede medir por: } \\
\text { - Costo menos depreciación acumulada menos deterioro acumula do. } \\
\text { - Valor de mercado, costo de reposición }{ }^{1 /} \text { o costo de reposición a nuevo depreciado } \\
\text { (Avalúo técnico). } \\
\text { - Valor actualizado en un período anterior, siempre y cuando se comparable con el valor } \\
\text { de mercado o con el costo depreciado. }\end{array}$ \\
\hline $\begin{array}{l}\text { Bienes de uso } \\
\text { público }\end{array}$ & $\begin{array}{l}\checkmark \text { Son destinados para uso, goce y disfrute de la comunidad. } \\
\text { El Estado es el responsable de administrarlos, protegerlos, mantenerlos física y } \\
\text { financieramente. } \\
\checkmark \text { Son inalienables }{ }^{2} \text {, imprescriptibles }{ }^{3 /} \text { e inembargables }{ }^{4} \text {. } \\
\checkmark \text { Se reconocen componentes. } \\
\checkmark \text { La medición inicial se realiza por el costo. } \\
\text { La medición posterior se realiza por el costo menos la depreciación acumulada menos el } \\
\text { deterioro acumulado. } \\
\text { Para la transición se puede medir por: } \\
\text { - Costo menos depreciación acumulada menos deterioro acumulado. } \\
\quad \text { Costo de reposición a nuevo depreciado (Avalúo técnico) }\end{array}$ \\
\hline
\end{tabular}

Fuente: elaboración propia a partir de CGN (2015a y 2015b).

1 / "Corresponde a la contraprestación más baja requerida para reemplazar el potencial de servicio restante de un activo o los beneficios económicos incorporados a este”. (Contaduría General de la Nación, 2015c, p. 16).

$2 /$ No se puede vender o enajenar.

3/ No se extinguen los derechos.

4/ No es posible embargarlos.

Los bienes de uso público se caracterizan por ser bienes de no mercado en los cuales su valor es inferior al valor de comercial y en algunas ocasiones su transferencia se lleva a cabo sin ninguna contraprestación. Dichas circunstancias dificultan la aplicación fiable de criterios de valoración debido a que plantea una restricción importante y es la ausencia de mercado, característica que no es tan frecuente en las propiedades, planta y equipo. Es decir, los criterios entre los dos tipos de activos coinciden mayoritariamente, sin embargo, es más difícil determinarlos para los bienes de uso público, especialmente para la transición, donde los costos pueden estar subvalorados o inclusive ser iguales a cero cuando los activos son antiguos o cuando son el resultado de una cesión urbanística.

A partir de este tipo de inconvenientes, y en especial debido a la carencia de metodologías de rigurosas técnicamente en el ámbito contable, se crea la necesidad de valorar dichos bienes, para esto, y con un trabajo empírico, se toman algunas teorías con el fin de desarrollar un modelo de valoración. Para la teoría económica el mejor asignador de recursos es el mercado, basados en aspectos de eficiencia y más bajo el supuesto de competencia perfecta, en donde los agentes económicos (consumidores, productores, trabajadores, etc.) toman decisiones de manera racional y con información completa, y en la interacción de los mismos (oferta y demanda) se configuran unos precios y unas cantidades de equilibrio, pero esta interacción no se cumple 
en los bienes de uso público, ya que dada su naturaleza se encuentran por fuera del mecanismo de formación de precios (Rojas, 2007).

Dada la imposibilidad de asignación de precio para los bienes de uso público se hace necesaria una valoración económica, es decir, "una herramienta que se utiliza para cuantificar, en términos monetarios, el valor de los bienes y servicios, independientemente de si estos cuentan o no con un precio o mercado" (Ministerio del Ambiente, 2015, p. 24). El objetivo de dicha valoración es analizar los beneficios que generan ante los cambios en la disponibilidad de espacio público, es decir la máxima cantidad de dinero que un individuo está Dispuesto A Pagar (DAP) para acceder a un cambio favorable en dicho bien.

Para dar cumplimiento al objetivo planteado el artículo incluye los siguientes apartados: la metodología, detallando los métodos y la fuente de información; posteriormente se presentan los resultados de la propuesta de medición para bienes de uso público, con el caso aplicado al Parque el Virrey, y por último, se plantean algunas ideas de conclusión y unas posibles líneas de investigación futuras.

\section{Metodología de los Precios Hedónicos}

La metodología de los precios hedónicos se usa para evaluar los valores económicos de un "ecosistema" que afectan directamente al mercado. Esta metodología fue formalizada e impulsada por Sherwin Rosen, estableciendo las bases teóricas más generales y proponiendo las bases conceptuales de la metodología (Riera, 1994, citado en Disegni, 2005; Azqueta, 1994; Aguilera, 2000). Generalmente, este método se aplica en el precio de las casas que refleja el valor de las características ambientales a su alrededor; esto siendo reflejo de la calidad ambiental (contaminación del aire, del agua e incluso el ruido), amenidades ambientales (vistas estéticas y cercanía de lugares recreacionales).

El trabajo de Edwin Causado, Rafael Viana y Oscar Jiménez (2008), trata de cómo los diferentes atributos determinantes del precio de mercado de los terrenos en los predios aledaños a Puerto Zúñiga; instalaciones donde ejerce su actividad carbonífera la empresa de C.I. PRODECO S.A., en las áreas de Pozos Colorados, Bello Horizonte y Don Jaca de la ciudad de Santa Marta están siendo afectadas por problemas referentes al deterioro ambiental (detrimento de la calidad del aire, deterioro de infraestructura, etc.), causando posibles pérdidas de bienestar de la población (Ministerio del Medio Ambiente, 2000). Las variables que utilizaron fueron: superficie del terreno (M2), área construida (M2) y estrato socioeconómico de los terrenos de Pozos Colorados, Bello Horizonte y Don Jaca de la ciudad de Santa Marta; datos obtenidos a través de una base de datos suministrada por Recaudos y Tributos S.A.

Tyrväinen y Mettinen (2000) muestran que en Salo, Finlandia, se paga un 4,8\% más por una vivienda que tenga vistas a un bosque, y cómo la proximidad a un parque forestal afecta al precio de la vivienda pues cada kilómetro que lo aleje del bosque o parque reduce el precio de ésta en un 5,7\%. Por su parte Bond, Seiler y Seiler (2002), hicieron una aproximación de cuánto aumenta el valor de una casa que tenga vista al lago Erie en Cleeveland, EE.UU. Muestran que una casa con vista al lago cuesta 89,9\% más que una casa que carezca de éste.

Des Rosiers (2002) estudia el impacto que las conexiones eléctricas tienen sobre el precio de las viviendas en Brossard, cerca de Montreal, Canadá. Concluye que el impacto visual de la vista directa de una torre de alta tensión tiene un impacto negativo sobre el valor de la vivienda con una reducción del valor aproximada de un 10\%. En este mismo contexto Wilhelmsson (2000) investiga el impacto que tiene el ruido procedente del tráfico sobre el valor de viviendas en Estocolmo, Suecia. Los resultados obtenidos señalan que en promedio por cada decibelio de más, el precio de la vivienda se reduce un $0,6 \%$, mientras que una casa situada en un lugar ruidoso vale un $30 \%$ menos que otra situada en un lugar un poco más silencioso.

De lo anterior se puede concluir que entre menos estén expuestas las viviendas o predios a los factores que afecten, modifiquen o intervengan en el paisajismo y el medio ambiente su valor comercial es superior que 
aquellos que se vean inmersos en este tipo de situaciones (Gutiérrez \& Díaz, 2017). Otra conclusión es que aquellos predios situados cerca o en nacimiento de aguas van a ser mucho más costosos por los escases de este recurso a nivel mundial.

\section{Metodología y resultados}

El enfoque utilizado es cuantitativo, por cuanto realiza medición y utiliza la estadística, además parte de un proceso deductivo. El estudio es exploratorio ya que se acoge a una perspectiva innovadora en el tema de estudio abriendo posibles líneas de investigación posterior y dando soluciones en un tema actual, como lo es proporcionar alternativas de medición de los bienes de uso público. Adicionalmente se utiliza el estudio de caso con el fin de ejemplificar la técnica propuesta en un bien específico, el cual corresponde al parque El Virrey en Bogotá.

El trabajo se realizó en el Grupo de Estudios y Análisis de la Defensoría del Espacio Público de Bogotá siguiendo estas etapas:

1. Se revisó la teoría económica y se eligió utilizar la metodología de los precios hedónicos.

2. Se determinaron las variables que influyen en la formación del precio del mercado edificado, y se procedió a hacer la recolección cartográfica, mediante Infraestructura de Datos Espaciales para el Distrito Capital (IDECA). Una vez recolectada y depurada la información se hizo el cálculo de la distancia Manhattan de cada manzana de la ciudad a las diferentes variables con el programa ArcGIS.

3. Se utilizó la relación dada por Box \& Cox (1964) con el fin de definir la forma funcional del modelo y su posterior ejecución utilizando el programa Stata 14.

4. Se determinaron las áreas de influencia de los diferentes espacios públicos usando el programa ArcGIS.

5. Se realizó la medición para el Parque El Virrey contrastando los datos obtenidos por la metodología con los datos arrojados por la Unidad Administrativa espacial de Catastro Distrital (UAECD).

Para determinar los valores de los diferentes espacios públicos existen diversos métodos como lo son valoración contingente, métodos de costos evitados, método de costo de viaje, entre otros. No obstante, dada su aplicabilidad y concordancia, este trabajo abordará la valoración desde la óptica de los precios hedónicos; este método permite valorar indirectamente los espacios públicos mediante la desagregación de los atributos que conforman dicho bien, este método parte de que lo que se compra genera una satisfacción, y dicha satisfacción se deriva de las características que conforman el bien.

Este método ha venido tomando importancia en la valoración de bienes de no mercado, pero existen contradicciones sobre quién fue el creador de la base de los precios hedónicos, ya que algunos autores atribuyen a Zvi Griliches como el primero en iniciar con este método a principios de los años sesenta. No obstante Allen Goodman, alude a Andrew Court el término "hedonic", ya que manifiesta el uso de este término por Court en un artículo en 1939. Pero de lo que no existe duda es que fue Rosen (1974) fue quien popularizó dicho método. (Gutiérrez \& Díaz, 2017, p. 10)

El método planteado busca estimar los valores económicos de bienes que carecen de mercado, mediante el análisis del precio de un bien de mercado, en este caso, un bien raíz en función de un conjunto de atributos (características estructurales de la vivienda, características socioeconómicas y características ambientales de la vivienda o el predio), es decir, dicho método permite derivar las demandas implícitas mediante técnicas econométricas,

permitiendo identificar la importancia relativa de cada atributo en el valor asignado por el mercado a un bien raíz, mediante lo cual es posible determinar cómo cambiará dicho valor al variar la cantidad y calidad en que se encuentra presente cada uno de estos atributos, y consecuentemente, predecir precios. (Lever, 2009, p. 3) 
Con el fin de derivar dichas demandas, es necesario colocar el precio del bien raíz en función de las características que conforman dicho bien, por lo cual la forma general del modelo utilizado es la siguiente:

$$
P=f(I, V, U, Z, S, E ; w)
$$

Dónde:

$P$ : precio del bien raíz.

I: características inherentes del bien raíz. Tales como el área construida, número de baños y acabados por ejemplo.

$V:$ características del vecindario. Tales como estrato, seguridad, entre otras.

$U$ : características de ubicación del bien raíz. Tales como las distancias a sitios de interés y/o equipamientos, como parques, centros comerciales, etc.

$Z$ : características determinadas por la ubicación del inmueble dentro del Plano Regulador de la ciudad (zoning), como los tipos de usos y diferentes densidades (empleo, construcción, población, entre otras).

$S$ : características determinadas por el nivel de equipamiento exterior, servicios e infraestructura que recibe el inmueble. Tales como los servicios que posee y el nivel de transporte, entre otros.

$E$ : externalidades presentes en el entorno en que se encuentra el bien raíz. Tales como el ruido, contaminación, parques y zonas verdes, entre otros.

$w$ : conjunto de parámetros que acompañan a cada atributo y que constituyen los precios implícitos (sombra) de cada característica del inmueble.

Dado que la relación de cada atributo con el precio del bien no presenta una relación lineal y basados en la evidencia empírica asociada al trabajo adelanto por Box \& Cox (1964) el cual permite estimar la relación funcional mediante la ecuación (2):

$$
P a=c+a X a+b Y a+d Z a
$$

Podemos determinar que la forma funcional para este tipo de modelos es muy aproximada a una función logarítmica por lo cual el modelo a estimar para la ciudad de Bogotá es el siguiente:

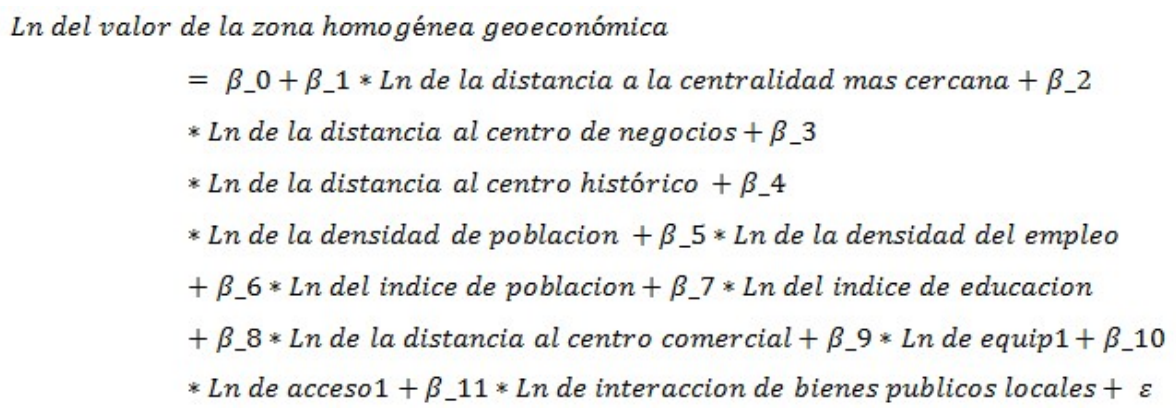

Donde equipl corresponde a la primera componente principal de las diferentes distancias a los equipamientos y accesol corresponde a la primera componente principal a las distancias que determinan el acceso a la manzana; la técnica de la primera componente principal: 
permite resumir la información contenida en las variables y facilitar su análisis. El ACP transforma un conjunto de variables originales en un conjunto de variables no correlacionadas denominadas componentes principales resultado de la combinación lineal de las variables originales hasta que se capture toda la variabilidad de la información (Araque, 2009, citado por Gutiérrez \& Díaz 2017).

En la tabla 2 se presenta el resumen de la construcción de las variables.

TABLA 2

Construcción de variables equip1 y accesol

\begin{tabular}{|l|r|}
\hline ACP & \multicolumn{1}{l|}{ equipl } \\
\hline Variable & Componente 1 \\
\hline distancia_abas & 0,3035 \\
\hline distancia_equip $\sim$ s & 0,3799 \\
\hline distancia_eq_curo & 0,4185 \\
\hline distancia_eq_cult & 0,3844 \\
\hline distancia_eq_educ & 0,3060 \\
\hline distancia_rec_fer & 0,1821 \\
\hline distancia_eq_re $-p$ & 0,2189 \\
\hline distancia_eq_sa $\sim d$ & 0,3936 \\
\hline distancia_eq_seg & 0,3332 \\
\hline
\end{tabular}

\begin{tabular}{|l|r|}
\hline ACP & accesol \\
\hline Variable & Componente 1 \\
\hline distancia_art & 0,3672 \\
\hline distancia_sitp & 0,5964 \\
\hline distancia_parad & 0,6156 \\
\hline dis_est_tm & 0,3614 \\
\hline
\end{tabular}

Fuente: elaboración propia.

Como lo indica el modelo, cuanto mayor es la distancia a los equipamientos (abastecimiento, bienestar social, de culto, de cultura, educación, recintos feriales, recreativos y deportivos, de salud y de seguridad) la componente principal (equip1) será mayor, análogamente se presentan los resultados para accesol la cual se encuentra asociada a las distancias a las vías articuladas, a las paradas del Sistema Integrado de Transporte (SITP), a las paradas de buses y a la estación del Sistema de transporte masivo de Bogotá (Transmilenio) más cercana.

Determinadas las variables y el modelo a estimar se realiza el análisis de regresión con el programa, obteniendo los siguientes resultados:

Número de observaciones $=24.104$

$\mathrm{F}(11,24092)=2.443,75$

Prob $>F=0,0000$

R-squared $=0,6532$

Root MSE $=0,38322$ 
TABLA 3

Coeficientes y errores de las variables

\begin{tabular}{|l|c|c|}
\hline Variable & Coeficiente & Error estadístico \\
\hline Ln_distancia_centralidad*** & $-0,0141124$ & 0,0020079 \\
\hline Ln_distancia_13*** & 0,2724089 & 0,0062345 \\
\hline Ln_distancia_72*** & $-0,365515$ & 0,0084824 \\
\hline Ln_densidad_poblacion*** & $-0,1971986$ & 0,0075424 \\
\hline Ln_densidad_empleo**** & 0,0354259 & 0,002754 \\
\hline Ln_indice_poblacion**** & $-0,3646422$ & 0,0385453 \\
\hline Ln_indice_educa*** & 0,5323943 & 0,0368032 \\
\hline Ln_distancia_centrocomercial**** & $-0,1506709$ & 0,0050365 \\
\hline Ln_equip1*** & $-0,1262351$ & 0,0101982 \\
\hline Ln_acceso1*** & $-0,0383143$ & 0,0111056 \\
\hline Ln_interaccion_bp1*** & $-0,0081116$ & 0,0007108 \\
\hline _cons*** & 18,02714 & 0,1139804 \\
\hline
\end{tabular}

Fuente: elaboración propia

${ }^{* * *} \mathrm{p}<0.01,{ }^{* *} \mathrm{p}<0.05,{ }^{*} \mathrm{p}<0.1$ corresponde al grado de significancia, en

este caso en particular todas las variables generaron una significancia del $99 \%$.

Como se observa en la salida de la regresión las variables "Ln_distancia_centralidad", "Ln_distancia_72", "Ln_distancia_centrocomercial", "Ln_interaccion_bpl" (la cercanía a la calle 72, distancia a centros comerciales y distancia a los bienes de uso público locales; variables establecidas como relevantes en el modelo.), "Ln_equip1", "Ln_acceso1”, que están en términos de distancias, presentan un coeficiente con signo negativo y dado que las variables se encuentran en términos de logaritmo natural se interpretan como la elasticidad precio de la distancia del bien, es decir en el caso de "Ln_distancia_centralidad" el cual su coeficiente es -0.0141124 , si incremento la distancia de 100 a 200 metros de la manzana a la centralidad, es decir un $100 \%$, el precio del suelo disminuye en un $1,4 \%$, con respecto al signo positivo que arroja la variable “Ln_distancia_13", que representa la distancia al centro histórico, este indica que la dinámica urbana, da prevalencia a las localizaciones que no se encuentran en cercanías a dicho centro, dado el alto índice de inseguridad que presenta la zona.

Con respecto a las variables "Ln_densidad_empleo" "Ln_densidad_poblacion", "Ln_indice_poblacion", y "Ln_indice_educa", sus coeficientes indican que en las partes de la ciudad donde se concentran las mayores densidades de población y empleo, como de los lugares donde se concentra la población adulta presentan mayores precios del suelo, al igual como en las partes de la ciudad donde se presentan los mayores índices de educación, el cual funciona como una proxy del ingreso, es decir a mayor nivel educativo, mayor ingreso y mayor capacidad de pago.

\section{Caso aplicado al Parque el Virrey}

Con el fin de mostrar un ejemplo práctico de la metodología adelantada se seleccionó para su aplicabilidad el Parque el Virrey. 
El Parque el Virrey se encuentra construido sobre un tradicional espacio verde del norte de Bogotá, y es uno de los primeros parques de carácter lineal debido a su extensión a lo largo del canal rio negro, el escenario se extiende varios kilómetros en sentido oriente y occidente entre la carrera Séptima y la autopista norte sobre la calles 87 y 88 . (Instituto Distrital de Recreación y Deporte, s.f.)

Para su aplicabilidad fue necesario determinar las áreas de impacto del parque con el fin de determinar los pagos que se efectúan por línea, por lo cual se construyeron 5 buffer ${ }^{1}$ con respecto al polígono del parque cada $100 \mathrm{mts}$ (ver figura 1), dentro de cada buffer se asignaron las manzanas las cuales pertenecían a dicha línea y fueron determinados los pagos acorde con la elasticidad precio distancia obtenida por el modelo de regresión.

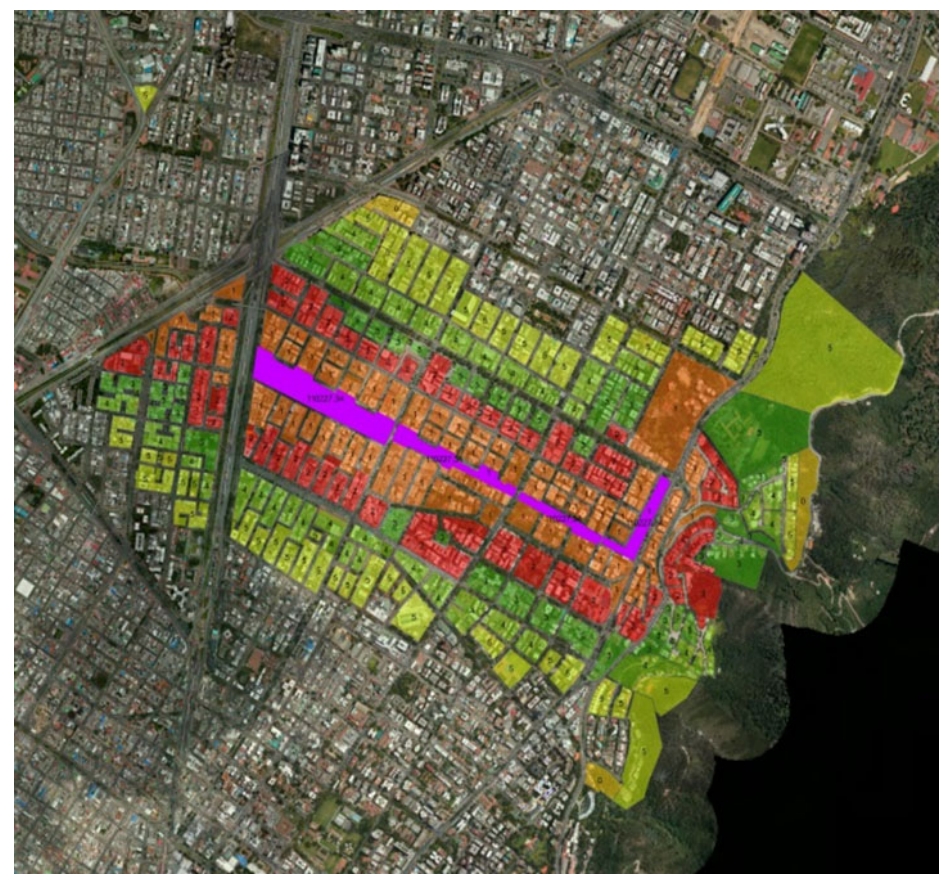

Figura 1. Orto foto del Parque El Virrey

Fuente: elaboración propia

En la imagen se observa el polígono central, el parque y las manzanas que colindan con el parque y su área de influencia de 500 metros.

En la tabla 4 se muestran las características del Parque El Virrey, el cual cuenta con un área de 21.227 metros cuadrados y tiene un avaluó catastral de \$1.877.509.400 lo que arroja un valor del metro cuadrado de $\$ 88.449$ monto muy inferior a la dinámica urbana del sector que cuenta con avalúos catastrales superiores a los dos millones y precios comerciales por encima de los 6 millones por metro cuadrado. Es importante resaltar como la metodología logra equiparar el valor del suelo público al valor del suelo privado, aspecto fundamental en la representación del costo de oportunidad del suelo, esto se evidencia en la razón inicial que existía entre el precio del suelo privado y el suelo público la cual era de 10 a 1 y con la metodología se estima una razón de 1 a 1.08. El parque tiene un valor de $\$ 64.441 .673 .759$ utilizando la metodología de precios hedónicos frente a $\$ 1.877 .509 .400$ por valor catastral. 
TABLA 4

Resultados de la valoración del parque el Virrey utilizando la metodología propuesta

\begin{tabular}{|l|r|}
\hline Concepto & \multicolumn{1}{|c|}{ Medida } \\
\hline Area del parque & $21.227 \mathrm{~m} 2$ \\
\hline Avalúo catastral & $\$ 1.877 .509 .400$ \\
\hline Avalúo $\mathrm{m} 2$ Terreno & $\$ 268.639$ \\
\hline Avalúo $\mathrm{m} 2$ Catastral & $\$ 88.449$ \\
\hline Avalúo m2 privado & $\$ 2.800 .513$ \\
\hline Valor metodología & $\$ 64.441 .673 .759$ \\
\hline Avalúo m2 metodología & $\$ 3.035 .835$ \\
\hline Razón inicial m2EP/m2Priv & 0,1 \\
\hline Razón estimada m2EP/m2Priv & 1,08 \\
\hline
\end{tabular}

Fuente: elaboración propia

La marcada diferencia se da gracias a que en la ciudad de Bogotá no existe ninguna entidad encargada de valorar los bienes de uso público, ya que la unidad delegada para realizar los avalúos de los predios de la ciudad tiene un papel fiscal, razón por la cual ha desarrollado metodologías de valoración muy avanzadas, pero estas, solo son aplicables para los predios privados dejando de lado los bienes de uso público, lo que ha generado que las entidades de gobierno que poseen bienes de uso público, no cuenten con la información necesaria para incluirla en sus estados financieros, lo cual ha contribuido a la desinformación tanto para los reguladores como para los entes privados. Por esto resulta de gran importancia contar con metodologías alternativas que nos brinden un acercamiento al verdadero valor de este tipo de bienes, con el fin de lograr una mejor información, administración y defensa de los mismos.

\section{Conclusiones}

El nuevo Régimen de Contabilidad Pública colombiano vinculó cambios sustanciales en el proceso contable de las diferentes entidades a nivel nacional y en específico en aquellas consideradas de gobierno. Uno de los efectos más significativos está relacionado con los criterios de medición que trae el reciente marco normativo contable, en especial la valoración de los bienes de no mercado que por sus características carecen de criterios fiables para su determinación. La posibilidad de poder hallar un valor preciso para estos bienes, con las limitantes que el nuevo modelo contable plantea, requiere que se utilicen fuentes metodológicas y teóricas que otras ciencias sociales, como la economía. Esto conlleva a la fijación de un valor adecuado para los bienes que carecen de mercado, lo que permite suministrar información financiera debidamente actualizada y a su vez será pertinente para los fines que los usuarios de dicha información requieran.

Con este escenario, se desarrolló una herramienta de medición basada en el diseño empírico de un modelo econométrico, el cual logra emplearse como estudio de caso a un parque público de la ciudad de Bogotá y puede replicarse con cualquier bien de estas características. Este análisis permite evidenciar, además, que el valor de los bienes de uso público reflejado en los estados financieros de las entidades de gobierno -en los casos que tenga algún valor asignado- difiere sustancialmente con la aplicación de la metodología, lo cual logra impactar la información financiera suministrada de manera positiva. 


\section{Referencias}

Aguilera, M. (2000). Perfil socioeconómico de Barranquilla, Cartagena y Santa Marta, 1990-2000. Documentos de Trabajo Sobre Economia Regional, No 17, Cartagena: Observatorio del Caribe Colombiano.

Azqueta O., D. (1994). Valoración económica de la calidad ambiental. Madrid: McGraw-Hill.

Box, G., \& Cox, D. (1964). An Analysis of Transformations. Journal of the Royal Statistical Society. Series B (26), 211-252. https://doi.org/10.1111/j.2517-6161.1964.tb00553.x

Causado, E., Viana, R., \& Jiménez, O. (2008). Estimación de un modelo hedónico para el precio de los predios en las áreas de Pozos Colorados, Bello Horizonte y Don Jaca de la ciudad de Santa Marta DTCH, Colombia. Clío América, 2(3), 99. http://dx.doi.org/10.21676/23897848.356

Contaduría General de la Nación. (junio de 2013). Estrategia de convergencia de la regulación contable pública hacia normas internacionales de información financiera (NIIF) y normas internacionales de contabilidad del sector público (NISP). Obtenido de http://www.contaduria.gov.co/wps/wcm/connect/8b13elfa-c7c9-4079-8f1d-9588d5d44f4a/Estrategia+d e+convergencia + de + la + regulaci\%C3\%B3n+contable + p\%C3\%BAblica + hacia + NIIF $+y+N I C S P . p d f ? M O D$ $=$ AJPERES

Contaduría General de la Nación. (08 de 10 de 2015a). Anexo Resolución 533 de 2015 Normas para el Reconocimiento, Medición, Revelación y Presentación de los Hechos Económicos de las entidades de gobierno. Bogotá, Colombia: Contaduría General de la Nación.

Contaduría General de la Nación. (08 de 10 de 2015b). Instructivo Nº 002, Instrucciones para la transición al Marco normativo para emtidades de gobierno. Bogotá: Contaduría General de la Nación.

Contaduría General de la Nación. (08 de 10 de 2015c). Marco Conceptual para la Preparación y Presentación de Información Financiera de las entidades de gobierno. Bogotá, Colombia: Contaduría General de la Nación.

Contaduría General de la Nación. (2 de 12 de 2015d). Referente teórico y metodológico de la Regulación Contable Pública. Bogotá: Contaduría General de la Nación.

Des Rosiers, F. (2002). Power lines, visual encumbrance and house value: A microspatial approach to impact measurement. Journal of Real Estate Research, 23, 276-330.

Disegni, N. (2005). Estimación de la demanda del agua en la Cuenca del Aconcagua a través de la metodología de Precios Hedónicos. Tesis para Optar al Grado de Magíster en Economía Agraria - Santiago. Pontificia Universidad Católica de Chile.

Gutiérrez, J., \& Díaz, M. (2017). Bogotá y el modelo de localización urbana. Cuadernos Latinoamericanos de Administración, 13(24), 9-17. http://dx.doi.org/10.18270/cuaderlam.v13i24

Hernández, R., Callado, C., \& Baptista, P. (2010). Metodología de la investigación. México DF: Mc Graw Hill.

Instituto Distrital de Recreación y Deporte. (s.f.). Parque El Virrey Norte. Recuperado el 30 de junio de 2016, de htt p://www.idrd.gov.co/sitio/idrd/?q=node/300

Lever, G. (2009). El modelo de los precios hedónicos. Recuperado el 30 de junio de 2016, de http://www.ucipfg.com /Repositorio/MAES/PED/Semana4/PreciosHedonicos.pdf

Ministerio del Ambiente. (2015). Guia Nacional de valoración económica del patrimonio natural. Lima: Harold Almonacid Flores.

Ministerio del Medio Ambiente. (2000). Guia Ambiental de Puertos Carboniferos. Bogotá.

Pérez, J., \& Merino, M. (s.f.). Definición. Recuperado el 05 de julio de 2016, de http://definicion.de/buffer/

Rico, C., \& Díaz, M. (2017). ¿Convergencia de la regulación contable colombiana para las entidades de gobierno con las International Public Sector Accounting Standards (IPSAS)? Una medición de la armonización formal. Contabilidady Negocios, 12(24), 61-83. https://doi.org/10.18800/contabilidad.201702.004

Riera, P. (1994). Manual de Valoración Contingente. Madrid: Editorial Civitas.

Rojas, J. (2007). Espacio "privatizado". El valor de privatizar un espacio público en zona urbana. Cataluña: Universitat Politècnica de Catalunya. 
Tyrväinen, L., \& Miettinen, A. (2000). Property prices and urban forest amenities. Journal of environmental economics and management, 39(2), 205-223. https://doi.org/10.1006/jeem.1999.1097

Wilhelmsson, M. (2000). El impacto del ruido del tráfico en los valores de casas unifamiliares. Revista de planificación y gestión ambiental, 43(6), 799-815. http://dx.doi.org/10.1080/09640560020001692

Zorrilla, A. (2012). Aplicación de la metodología de precios hedónicos para la valoración ambiental de las áreas verdes urbanas en la ciudad de Bogotá. Bogotá: Repositorio Universidad Santo Tomás.

\section{Notas}

1 "El polígono que enmarca el área de influencia que resulta al tomar una determinada distancia desde un punto, línea u otro polígono" (Pérez \& Merino, s.f.)

*Artículo de investigación científica y tecnológica.

\section{Licencia Creative Commons CC BY 4.0}

Para citar este artículo: Díaz J., M. A., Gutiérrez L., J. A. \& Patiño J., R. A. (2018). Valoración de bienes de uso público en el modelo contable de entidades de gobierno en Colombia. Una aproximación desde los precios hedónicos. Cuadernos de Contabilidad, 19(48), 1-12. https://doi.org/10.11144/Javeriana.cc19-48.vbup 